\title{
The electroretinogram in minimal diabetic retinopathy
}

\author{
T C A Jenkins, J P Cartwright
}

\begin{abstract}
The pattern and diffuse flash electroretinograms were measured in 20 normal subjects and 40 diabetic patients who had either normal fundi or microaneurysms only. The amplitudes of the pattern electroretinogram were found to be similar in both normals and diabetics. In the case of the flash electroretinogram the diabetic patients showed a division into two main groups. One group was not dissimilar to the group of normal subjects, while the second group showed hypernormal amplitudes. No explanation could be given, from the data collected, for this subdivision, though it is suggested it might reflect the degree of metabolic disturbance.
\end{abstract}

We have recently been connected with work that involves the monitoring of visual changes in eyes with minimal diabetic retinopathy. The battery of tests involved included fundus photography and a number of vision assessments such as colour vision, contrast sensitivity, and critical fusion frequency. It did not, however, include initially any electrodiagnostic test, and this paper arises from an assessment as to whether or not electroretinography would be a useful addition.

Several features of the electroretinogram (ERG) have been studied in diabetic retinopathy. Perhaps the oscillatory potentials (OPs) have given rise to more reports than any other. There seems to be no doubt that they are affected at some stage, but it is more likely that it is in the later preproliferative stages that they are most seriously affected ${ }^{1-3}$ and that actual prognostic evaluation concerning proliferative development is not insignificant. ${ }^{46}$ Coupland ${ }^{7}$ suggests that there is not only a reduction of OP amplitude below normal in diabetic patients with background retinopathy but also in those with no background retinopathy. However, Arden et al suggest that in individual patients OP amplitude is not a particularly good indicator of the disease's progress, though they agree that there is a general deterioration in the OPs with severity. Lovasik and Spafford ${ }^{9}$ investigated 30 young insulin dependent patients with normal retinal vasculature and found significant differences, but they were so small that they were not considered diagnostic.

Amplitudes of the diffuse flash ERG, in themselves, have not been as widely studied. Certainly as a reflection of receptor activity ${ }^{10}$ it would not be surprising if they were affected. Perlman" points out that the wave can be taken to represent the input to the proximal retina and the $b$ wave its output. However, most studies concern themselves with very late stages of retinopathy and deal with retinal function before and after photocoagulation. ${ }^{12-1+}$ Gjotterberg' noted an interesting tendency to hypernormal amplitudes in background retinopathy, whereas those at the proliferative stage showed subnormal values. He makes the point that striving for conditions to produce maximum amplitudes is not necessarily best. The problem with an intense light flash is that, although very large waves can be produced, the dispersion of light within the eye, even in normals, occurs in an unpredictable fashion. In the present work we deliberately chose a low flash intensity to attempt to keep intersubject variability, within normals, to a minimum.

It has been suggested ${ }^{10}$ that the pattern ERG (PERG) produced by a reversing black and white check pattern reflects ganglion cell activity, and, while it may be useful in diseases of the optic nerve and ganglion cell body, it may be less useful in diabetic retinopathy, particularly in minimal retinopathy. ${ }^{7}$ According to Arden et al ${ }^{8}$ a reduction in PERG amplitude appears at the phase of the disease when referral to an ophthalmologist is necessary. They suggest no difference occured between a group of diabetic patients with normal fundi and a group with microaneurysms only, though a difference-of-means test on their data suggests this might not have been conclusive. Because these gradings corresponded to those of the present study a PERG was undertaken as a confirmatory experiment.

\section{Patients and methods}

Two groups of subjects were invovled in the work and all gave their consent after being given an explanation of the nature of the experiments.

The diabetic group consisted of 40 non-insulin dependent patients who were passing through the clinics of the Bradford University diabetic visual screening programme. The age range was preset at 40-65 years, and all those involved had been diagnosed as diabetic for at least four years. The mean time was in fact $9 \cdot 9$ (range $4-28$ ) years. All patients had to have an acuity of at least $6 / 9$, corrected if necessary, and any eye condition other than diabetes excluded them. The possession of clear media was also a criterion for inclusion in the group.

At the beginning of the experiments full details of all patients were available, including non-stereoscopic photography. Fundus classification was based on a single photograph of the central $30^{\circ}$ rather than on a more extensive set of photographs, ${ }^{15}$ and patients were to have minimal retinopathy. Quite deliberately 20 were chosen with normal fundi (classed as level 10) and 20 with microaneurysms only (classed as level 20). The mean age of the total group was $54 \cdot 3$ (SD6.8) years, and the range reached the preset value of $40-65$ years. 
The normal group consisted of 20 people with no history of eye disease and who presented no abnormality on examination. A minimum level of 6/9 acuity was kept to as in the diabetic group, and as close an age match with the diabetic group was made as practicable within the stated age band. The mean age of the 20 subjects in this group was $52.6(\mathrm{SD6} \cdot 1)$ years, with the actual range 42-64 years.

A Nicolet CA 1000 clinical averager and Nicolet HGA 200A amplifier were employed together with a Nicolet NIC 1015 visual stimulator to provide the PERG stimulus. The check pattern reversal stimulus subtended a field size of $30^{\circ} \times 24^{\circ}$ and the individual check size was $30^{\prime}$. Toobtain this the television screen was positioned at $85 \mathrm{~cm}$ from the subject and a small fixation spot was used to help the maintenance of steady fixation. The luminance of the dark squares was $11 \mathrm{~cd} / \mathrm{m}^{2}$ and that of the light squares was $140 \mathrm{~cd} /$ $\mathrm{m}^{2}$, giving a contrast of $85 \%$.

A Grass PS 22 photic stimulator, at the lowest intensity of unity, was used to provide the flash stimulus which at a distance of $23 \mathrm{~cm}$ subtended a circular field of $30^{\circ}$. No fixation spot was provided, and the subject was simply requested to fix the centre of the light. The low intensity flash stimulus was employed in an attempt to contain the large degree of scatter that can take place in the eye when a high intensity stimulus is used.

For both the PERG and flash ERG the band pass filters were set at 1 and $50 \mathrm{~Hz}$ with the sensitivity at $\pm 100 \mu \mathrm{V}$. A stimulus presentation rate of 4 alternations per second and 4 flashes per second were used for both the PERG and flash ERG respectively. In both cases the analysis time was $150 \mathrm{~ms}$.

All runs were under photopic conditions, and
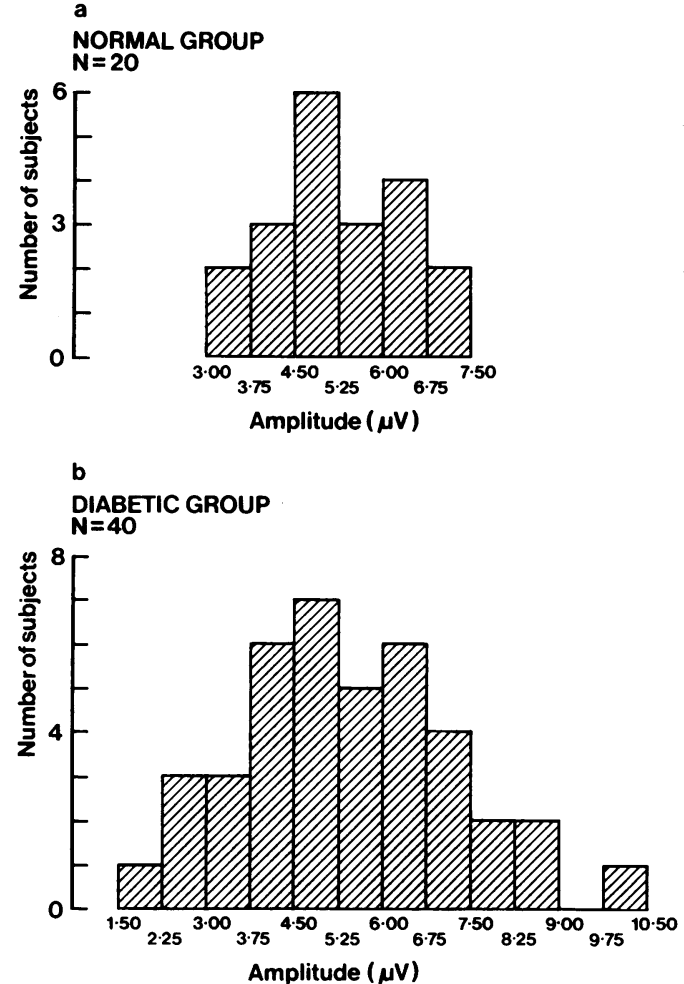

Figure 1 Histograms indicating the distribution of PERG amplitudes in (a) 20 normal subjects, (b) 40 diabetic patients. subjects had an adaptive period in the room of about 10 minutes. The ambient illumination in the plane of the eyes was approximately 150 lux.

The active electrode was DTL fibre, placed under the lower lid, which was referenced to an electrode at the centre of the forehead with a ground electrode to the side of the forehead. The maximum interelectrode impedance acceptable was $4 \mathrm{kohm}$.

The PERG was recorded first, and all subjects wore a distance correction if one was present. In the case of those over 50 years of age a +0.50 sphere was added, quite arbitrarily, to compensate for the near fixation distance. The wave produced was the averaged product of 360 sweeps, though they were recorded in six runs of 60 sweeps, each run being preceeded by a sixsecond fixation period. Inter-run rest periods of about 20-30 seconds were given. For convenience the right eyes of subjects were used, though in the case of three diabetic patients the left eye was used. Throughout the experiments the eye not under investigation was occluded. Pupils were undilated in all cases.

A rest interval was given of about 2 minutes before measurement of the flash ERG. In this case all readings were taken from the unaided eye. A 100-sweep wave was recorded in two runs of 50 sweeps. Again, a 6-second adaptive period was given before recording began and a single 20-30 second rest interval was provided between the two runs.

In the case of the PERG the amplitude was measured from the positive peak which occurs at 50-55 ms to the following trough which occurs at 95-100 ms (after Holder ${ }^{16}$ ). The flash ERG was recorded as the $b$ wave amplitude from the trough of the a wave to the peak of the $b$ wave.

\section{Results}

PATTERN ELECTRORETINOGRAM

Histograms indicating the PERG amplitudes for both the normal and diabetic groups are shown in Fig 1. Both sets of data appeared normally distributed when examined by a $\chi^{2}$ test for normality. The mean for the normal group was $5.25(\mathrm{SD} 1 \cdot 15) \mu \mathrm{V}$ and that for the diabetic was very close at $5.40(\mathrm{SD1} \cdot 85) \mu \mathrm{V}$. It will be seen in Fig 1 that the spread of results for the diabetic group appears greater than for the normal subjects. An $F$ test on the variances suggested $(F=$ $2.59, p=0.01$ ) that this was very likely to have been the case.

The individual diabetic group means were $5 \cdot 68$ (SD1 -57) $\mu \mathrm{V}$ for level 10 and 5.13 (SD2.06) $\mu \mathrm{V}$ for level 20 , though a $t$ test for unrelated data showed no significant difference between them. Hence there was no evidence that the mean values for the diabetic patients were different from that of a normal age matched group.

Mean latencies for the normals were 55.02 (SD3.59) msec and 99.28 (SD9.97) for the positive peak and proceeding negative trough respectively. For the diabetic group the values differed little, and certainly not statistically significantly so, at $\mathbf{5 4 . 5 0}$ (SD3.84) ms and $\mathbf{9 9 . 6 5}$ (SD8.55) ms.

It has been reported ${ }^{17}$ for the PERG that there 
is a small negative correlation between amplitude and age and a small positive one between the major positive peak latency and age. The former was confirmed here by a value of $r=-0.26(p=$ 0.05 ) for the total subject group of 60 people, though a not significant value, of $r=0 \cdot 18$, was found for the latter. The age spread in the present investigation was, however, relatively narrow.

\section{DIFFUSE FLASH ELECTRORETINOGRAM}

Fig 2 shows histograms for both the normal and diabetic groups. While $\chi^{2}$ tests for normality suggest the data of the normal subjects were normally distributed, that of the diabetic patients were not so distributed $(p=0 \cdot 01)$. The mean amplitude (trough of a wave to peak of $b$ wave) for the normal group was $36 \cdot 8$ (SD9.1) $\mu \mathrm{V}$. The diabetic distribution appears bimodal in character with the two main subgroups of $A$ and $B$ as indicated in the figure. The mean of the $\mathbf{4 0}$ diabetics was 40.5 (SD11.3) $\mu \mathrm{V}$, though clearly the use of a mean and standard deviation to describe such a distribution is very misleading. In Fig 2 such a value is very much in the trough of the distribution.

When the two main groups, A and B, were looked at in terms of a division between level 10 and level 20 patients, no relationship was found. It was certainly not true that those in group $\mathbf{A}$ were mainly level 10 patients and those in group $B$ level 20. Indeed the bimodal division occurred in both level 10 and level 20 patients to about the same extent.

The subdivision appeared unrelated to the patient's age or to the length of time diagnosed as diabetic. The correlation coefficient between
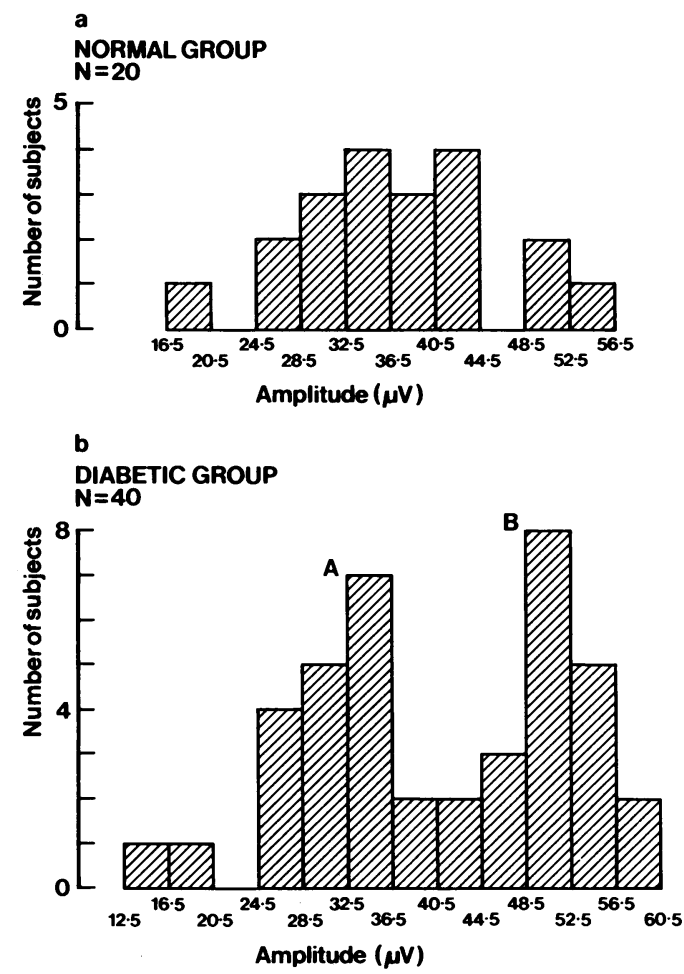

Figure 2 Histograms indicating the distribution of diffuse flash ERG b wave amblitudes in (a) 20 normal subjects (b) 40 diabetic patients. In the case of the diabetic patients the letters $A$ and $B$ indicate two subgroups. wave amplitude and length of time diabetic was, in fact, zero and that between amplitude and age was only $0 \cdot 05$.

Unfortunately, because of the impossibility of relating the subgroups to any known factor, it was impossible to decide on the exact dimensions of these subgroups. They appear perhaps appoximately equal in size, and group $A$ values look to be not very different from those of the normal subjects. At the other extreme, $45 \%$ of the total diabetic group had amplitudes over $44 \mu \mathrm{V}$.

The lack of correlation between amplitude and age held for the normal group $(r=0 \cdot 13$, not significant) as well as for the diabetic group, which suggests the small inverse relationship which appears to be present for the PERG does not hold for the flash ERG. There was, however, a small $(r=0.33, p=0.01)$ but statistically significant correlation between the PERG and flash amplitudes for the total group of 60 subjects. It remained at about this value for the normal group and level 10 and level 20 groups individually.

There was no evidence to suggest that the distribution of the $a$ or $b$ wave latencies in the diabetic group was other than a normal distribution, and there was little difference between the mean values for the normal and diabetic groups. For the former they were 20.67 (SD0.90) $\mathrm{ms}$ for the a wave and 39.87 (SD1.70) ms for the b wave and for the latter $20 \cdot 80(\mathrm{SDl} \cdot 27) \mathrm{ms}$ and 40.31 (SD2.65) ms respectively. Variance ratio tests suggested $(p=0.05$ for the a wave, $p=0.01$ for the $b$ wave) that there was more variability in the latencies in the diabetics than in the normals.

\section{Discussion}

The results of the PERG confirmed those of

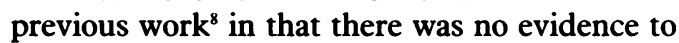
suggest the amplitudes of diabetics with minimal retinopathy tend to be any different from normal subjects. The values were only a little below those of Hull and Drasdo, ${ }^{17}$ who obtained a mean amplitude of 5.68 (SD1.94) $\mu \mathrm{V}$ on normals, though because their sample contained a number of young subjects this slight increase is not surprising.

In the case of the flash ERG there was no 'a priori' reason to suspect the identification of two main groups, and indeed it was not possible to relate such groups to factors such as age, length of time diabetic, or to retinopathy level. Some estimate may be made as to amplitude values that can be considered hypernormal from the data of the normal subjects. The normal mean was 36.8 (SD 9.1) $\mu \mathrm{V}$. For a normal distribution with such parameters $20 \%$ of subjects would be expected to have amplitudes of, or greater than, $36 \cdot 8+0 \cdot 84(9 \cdot 1) \mu \mathrm{V}$ that is, $44.5 \mu \mathrm{V}$. When the actual data were looked at, it was seen that three people $(15 \%)$ were so categorised and that this increased to $18(45 \%)$ of the diabetic patients. If a more rigorous, $5 \%$ cut off criterion is used, then the critical value becomes, $36 \cdot 8+1.65(9 \cdot 1) \mu \mathrm{V}$ that is, $52 \mu \mathrm{V}$. When applied to the experimental data, this excluded one normal $(5 \%)$ and seven $(18 \%)$ of the diabetics.

Gjotterberg' found a tendency to hypernormal 
amplitudes in a group of diabetics with background retinopathy and argued that, since there is evidence ${ }^{18}$ that such cases have an increased retinal blood flow, this would result in a higher ERG amplitude. It is tempting to try to interpret the present findings in such terms, though it is almost certainly too simplistic to see an increased amplitude necessarily equating to an increased blood supply.

The reason for hypernormality of amplitude in some diabetics might not even be a retinal one. Although the diabetics used in this study were defined as having clear media, it is uncertain how significant such changes would have to be to produce a dispersive effect sufficiently great to alter wave amplitude. We have carried out a study of the effect of using lightly frosted plano lenses before the eye when measuring the ERG. ${ }^{19}$ The mildest degree of frosting used was such as to produce a lens which looked slightly dirty, thought it reduced visual acuity on a group of 10 normal subjects by only two letters on the $6 / 4$ line. When a very low flash stimulus (unity on the Grass scale with a $712^{\circ}$ field size) was used, the $b$ wave amplitude increased by nearly $60 \%$ $(3.4 \mu \mathrm{V}$ to $5.4 \mu \mathrm{V})$ when compared to that of a clear plano lens. Clearly low dispersive effects can make a considerable difference to ERG results.

Recent work by U Dhanesha et al (personal communication, 1990), using a similar group of diabetics, has also produced bimodality in the data. They made psychophysical determinations of visual functioning and showed that the results divided into two main groups according to the degree of metabolic disturbance in the patients. It is tentatively suggested that the ERG findings described here might be a further indication of such a division.
1 Giotterberg $M$. The electroretinogram in diabetic retinopathy. Arch Ophthalmol (kbh) 1974; 52: 521-33.

2 Bresnick GH, Korth K, Groo A, Palta M. Electroretinographic oscillatory potentials predict progression of diabetic retinooscillatory potentials predict progression of
pathy. Arch Ophthalmol 1984;102: 1307-11.

3 Brinchmann-Hansen O, Dahl-Jorgensen $\mathrm{K}$, Hanssen KF, Brinchmann-Hansen $O$, Dahl-Jorgensen $K$, Hanssen $\mathrm{KF}$,
Sandvick L. Oscillatory potentials, macular recovery time Sandvick $L$. Oscillatory potentials, macular recovery time
and diabetic retinopathy through 3 years of intensified and diabetic retinopathy through 3 years of inte

4 Simonsen SE. Electroretinographic study of diabetics: preliminary report. Acta Ophthalmol $(\mathrm{kbh}) 1965 ; 43: 841-3$.

5 Simonsen SE Prognostic value of ERG (oscillatory potential) in juvenile diabetics. Acta Ophthalmol (kbh) 1975; 123 (suppl): 223-4.

6 Moschos M, Panagakis E, Augelopoulos A. Changes of oscillatory potentials of the ERG in diabetic retinopathy. Ophthalmic Physiol Opt 1987; 7: 477-9.

7 Coupland SG. A comparison of oscillatory and pattern electroretinogram measures in diabetic retinopathy. Doc Ophthalmol 1987; 66: 207-18.

8 Arden GB, Hamilton AMP, Wilson-Holt J, Ryan S, Yudkin JS, Kurtz A. Pattern electroretinograms become abnormal wh, Kurtz A. Pattern electroretinograms become abnormal when background diabetic retinopathy deteriorates to a preproliferative stage: possible

9 Lovasik JV, Spafford MM. An electrophysiological investigation of visual function in juvenile insulin-dependen diabetes mellitus. Am F Optom Physiol Opt 1988; 65: 236-53.

10 Maffei L, Fiorentini A. Electroretinographic response to alternate gratings before and after section of the optic nerve. Science 1981; 211: 953-5.

11 Perlman I. Relationship between the amplitudes of the $b$ wave and the a wave as a useful index for evaluating the electroretinogram. Brf Ophthalmol 1983; 67: 443-8.

12 François J, De Rouck A, Cambie E, Castanheira-Dinis A Electrophysiological studies before and after argon laser photocoagulation in diabetic retinopathy. Ophthalmologica 1978; 176: $133-44$.

13 Liang JC, Fishman GA, Huamonte FV, Anderson RJ. Comparative electroretinograms in argon laser and xenon arc parative electroretinograms in argon laser and xenon arc $520-5$.

14 Perlman I, Gdal-on M, Miller B, Zonis S. Retinal function of the diabetic retina after argon laser photocoagulation assessed. Br F Ophthalmol 1985; 69: 240-6.

15 Davis MD, Hubbard LD, Trautman J, Klein R. Studies of retinopathy. Methodology for assessment and classification with fundus photographs. Diabetes 1985; 34 (suppl 3): 42-9.

16 Holder GE. Significance of abnormal pattern electroretinography in anterior visual pathology dysfunction., $\mathrm{Br} \mathcal{F}$ Ophthalmol 1987; 71: 166-71.

17 Hull BM, Drasdo N. The influence of age on the pattern reversal electroretinogram. Ophthalmic Physiol Opt 1990; 10: 49-53.

18 Kohner EM. The effect of diabetes on retino-vascular function. Acta Diabetol Lat 1971; 8 (suppl 1): 135-78.

19 Douthwaite WA, Jenkins TCA, Gilmore DP. Electrophysiological investigation of simulated media opacities. Report (unpublished). University of Bradford: 1990. 